To be presented at Fall Meeting of the Materials Research Society, Boston, MA, December 1-5, 1997, and published in Proceedings:

\title{
CURRENT LOCALIZATION, NON-UNIFORM HEATING, AND FAILURES OF ZnO VARISTORS
}

\author{
M. Bartkowiak, \\ Solid State Division, Oak Ridge National Laboratory \\ Oak Ridge, Tennessee 37831-6030 \\ and \\ University of Tennessee, Dept. of Physics and Astronomy, \\ Knoxville, TN 37996-1200
}

"The submitted manuscript has been authored by a contractor of the US Goverment under contract No. DE-AC05-960R22464. Accordingly, the U.S. Government retains a nonexclusive, royalty-free license to publish or reproduce the published form of this contribution, or allow others to do so, for U.S. Government purposes."

RECEIVED

JAN 261998

OSTI

\author{
prepared by \\ SOLID STATE DIVISION \\ OAK RIDGE NATIONAL LABORATORY \\ Managed by \\ LOCKHEED MARTIN ENERGY RESEARCH CORP. \\ under \\ Contract No. DE-AC05-96OR22464 \\ with the \\ U.S. DEPARTMENT OF ENERGY \\ Oak Ridge, Tennessee

November 1997 


\section{DISCLAIMER}

This report was prepared as an account of work sponsored by an agency of the United States Government. Neither the United States Government nor any agency thereof, nor any of their employees, makes any warranty, express or implied, or assumes any legal liability or responsibility for the accuracy, completeness, or usefulness of any information, apparatus, product, or process disclosed, or represents that its use would not infringe privately owned rights. Reference herein to any specific commercial product, process, or service by trade name, trademark, manufacturer, or otherwise does not necessarily constitute or imply its endorsement, recommendation, or favoring by the United States Government or any agency thereof. The views and opinions of authors expressed herein do not necessarily state or reflect those of the United States Government or any agency thereof. 


\section{DISCLAIMER}

Portions of this document may be illegible electronic image products. Images are produced from the best available original document. 


\section{CURRENT LOCALIZATION, NON-UNIFORM HEATING, AND FAILURES OF ZnO VARISTORS}

\section{BARTKOWIAK}

Department of Physics and Astronomy, University of Tennessee, Knoxville, TN 37996-1200, and Solid State Division, Oak Ridge National Laboratory, Oak Ridge, TN 37831-6030, bartkowiakm@ornl.gov

\section{ABSTRACT}

Non-uniform heating of $\mathrm{ZnO}$ varistors by electrical pulses occurs on three different spatial scales: (1) microscopic (sub-micron), (2) intermediate (sub-millimiter), and (3) macroscopic (of order of millimeters or centimeters). Heating on these scales has different origins and different consequences for device failure in large and small varistors. On the microscopic scale, the heating localizes in strings of tiny hot spots. They occur at the grain boundaries in a conducting path where the potential is dropped across Schottky-type barriers. These observations are interpreted by applying transport theory and using computer simulations. It is shown that the heat transfer on a scale of the grain size is too fast to permit temperature differences that could cause a varistor failure. On an intermediate size scale, the heating is most intense along localized electrical paths. The high electrical conductivity of these paths has microstructural origin, i.e., it derives from the statistical fuctuations of grain sizes and grain boundary properties. Current localization on the intermediate size scale appears to be significant only in small varistors. On the macroscopic scale, current localization in large blocks can be attributed to inhomogeneities in the electrical properties which originate during ceramic processing. The resulting non-uniform heating is shown to cause destructive failures of large varistor blocks.

\section{INTRODUCTION}

Metal oxide varistors have highly nonlinear electrical characteristics and are widely used as devices for over-voltage protection $[1,2]$. Varistor applications range from the use of small varistors to protect delicate electronic components to the use of much larger varistors for the protection of electrical-power-distribution systems. Specifically, varistors provide protection against voltage surges due to lightning strikes, switching transients, and similar disturbances. While varistors have a large capacity to absorb energy (e.g., $500 \mathrm{~J} / \mathrm{cm}^{3}$ ), they are, in fact, subject to occasional failure. The significant varistor-failure mechanisms include: electrical puncture, thermal cracking, and thermal runaway - all resulting from excessive heating, in particular, from non-uniform heating. Temperature and current flow in a varistor are highly correlated, since the heat is generated by electrical conduction which varies strongly with temperature. Non-uniform Joule heating occurs in varistors as a result of inhomogeneous electrical properties that originate in either the varistor fabrication process or in the statistical fluctuations in properties that generally occur in polycrystalline materials.

The power dissipation characteristics, Joule heating, and energy-handling capability of 
$\mathrm{ZnO}$ varistors have attracted attention because of their relevance to device failure and service life [3]-[13]. Measurements have been made that directly connect varistor failure to heating, and the various modes of varistor failure have been modeled. Eda [3] has used simple electrical measurements to establish that non-uniform electrical properties cause varistor failures. In his work, a spatial variation of the breakdown voltage in large varistors was measured by placing pairs of small electrodes at various positions. Eda attributed this variation in the breakdown voltage to a broad grain-size distribution. Weak spots with low-breakdown voltage and high-current conduction were attributed to a lower number of grain boundaries present in current paths for materials containing large grains. (Recent evidence suggests that weak spots in small varistors also can result from a reduction in the barrier potentials as well as in the number of grains - i.e., grain boundaries may be present but electrically ineffective [14]-[18]). These highly conducting regions heat up more rapidly than their surroundings under high currents and generate thermal stresses that cause cracking. High current can also cause local heating sufficient to melt the $\mathrm{Bi}_{2} \mathrm{O}_{3}$ phase and, thereby produce so-called "puncture" failures. Alternatively, the temperature of a varistor may become so hot that a thermal runaway failure ensues due to the material's negative temperature coefficient of resistivity [4]-[7].

Non-uniform electrical conduction in varistors has been observed at a higher spatial resolution by using scanning contacts and galvanic electroplating [8], but such measurements are well suited only to low-current densities and they provide essentially no time resolution. Mizukoshi et al. observed non-uniform heating in varistors by infrared (IR) imaging [9] using an IR camera to image hot zones in station-class arresters, and they quantified the relationships between non-uniformity and varistor failures. But the spatial resolution and temperature resolution of these experimental methods were quite limited. Moreover, the IR images were obtained long after the electrical pulses, so no time resolution of the heating process was observed. In a recent study [10], a new, high-speed IR camera was utilized to monitor more precisely the heating transients occurring in varistors during the electrical breakdown process. Time resolved measurements with high spatial and high temperature resolution provided detailed information on varistor heating on both a macroscopic and a microscopic scale during and after the application of electrical transients. On a macroscopic scale, large arrester blocks exhibited non-uniform heating that is related to their ceramic processing. On an intermediate size scale, non-uniform heating in small varistor disks was found to be most intense along highly conducting current paths that result from the statistical fluctuations in electrical properties which occur in polycrystalline materials. On a microscopic scale, the heat generation in varistors was observed to be localized at the grain boundaries.

In principle, non-uniform heating on each of the above size scales may lead to a varistor failure. The important question how heating on these vastly different time and size scales relates to puncture or cracking has been addressed by applying transport theory and by using computer simulations. The results obtained from these calculations are in excellent agreement with the experimental data. It is shown that on a scale of the grain size the heat transfer is simply too fast to permit temperature differences that could cause a varistor failure. Equally interesting results have been obtained for the case of current localization on an intermediate size scale because of microstructural disorder. Recent results from computer simulations [15]-[18] show that current flowing through the disordered grain microstructure of varistors localizes almost completely in a few very narrow paths. Given these results, it may seem strange that puncture or cracking failure does not always occur! We have resolved 
this issue by showing that current localization of microstructural origin is important only in small varistors. The degree of current localization is indeed very high in varistors of the a size that can be easily simulated on a computer, but it becomes much less significant in larger varistors. On the other hand, puncture and cracking failures of large varistor blocks are caused by non-uniform current flow and non-uniform heating on a macroscopic scale that can be attributed to imperfect processing. Conditions under which these failures can occur are described in detail in references [6] and [7].

\section{MACROSCOPIC SCALE}

Experimental data on two different kinds of large $(4.2 \mathrm{~cm}$ diameter) varistor blocks are provided in Ref. [10]. One kind of varistors were fabricated with a $1 \mathrm{~cm}$ thickness, and the other were a $1 \mathrm{~cm}$ thick pieces cut from commercial blocks made with the same diameter but a $4.6 \mathrm{~cm}$ length. Both kinds of blocks were heated by a $10 \mathrm{~A}$ current pulse with a $1 \mathrm{~ms}$ duration and the time resolved thermal images of the surface temperature were recorded with an IR camera. For the case of the varistors fabricated with a $1 \mathrm{~cm}$ thickness, the circumference of the blocks represented the hottest region, and the temperature distribution was roughly radially symmetric. Localized heating in the blocks interior, as reported by Mizukoshi et al. [9], was not observed. However, for the $1 \mathrm{~cm}$ thick pieces cut from commercial blocks, nonuniform heating localized in hot spots were clearly visible, and the IR images were similar to those reported Mizukoshi et al. These results are consistent with the greater difficulty of fabricating uniform large blocks, particularly blocks with a high aspect ratio.

Thermal images of the varistors were analyzed to determine the temperature distribution in the block as a function of time. Temperature profiles along a disk diameter before, during, and after the current pulse were measured. A few of these profiles for a varistor block fabricated with a $1 \mathrm{~cm}$ thickness are shown in Fig. 1(a). An initial temperature profile was taken from an image made during the current pulse. It represents the temperature of the block at a time half way through the pulse, or at $0.5 \mathrm{~ms}$. During the current pulse, the temperature was rising very fast, particularly at the periphery of the block. But after the pulse, the temperature changed relatively slowly on the centimeter size scale of the block.

The temperature rises most at the periphery of the block because most of the current flow is taking place there. This inference was checked using Eda's approach of placing pairs of small electrodes at various positions on the block. A 3 to $4 \%$ decrease in the breakdown voltage from the center to the edge was measured directly. These measured values of the breakdown voltage have been expressed as an approximate analytical function of the distance from the center of the disk, and used to simulate its thermal behavior by solving numerically the coupled equations describing the heat transfer and the non-uniform flow of the nonlinear currents. We have assumed that the disk can dissipate heat only radially by convection through the sidewall, and used realistic temperature-dependent data for thermal properties of the material and for the varistor I-V characteristic. At temperatures close to room temperature, we have used: the heat capacity $c=0.6 \mathrm{~J} /(\mathrm{gK})$, the diffusivity $D=0.1 \mathrm{~cm}^{2} / \mathrm{s}$, and the density $\rho=5.5 \mathrm{~g} / \mathrm{cm}^{3}$. Details of the model are described in Ref. [6]. For the case of a $1 \mathrm{~ms}, 10 \mathrm{~A}$ current pulse, the temperature profiles of the disk have been calculated for the times corresponding to those in the measurements. The results are presented in Fig. 1(b). They are in very good agreement with the experimental data in Fig. 1(a).

Because the temperature distribution shown in Fig. 1(a) has radial symmetry of the 

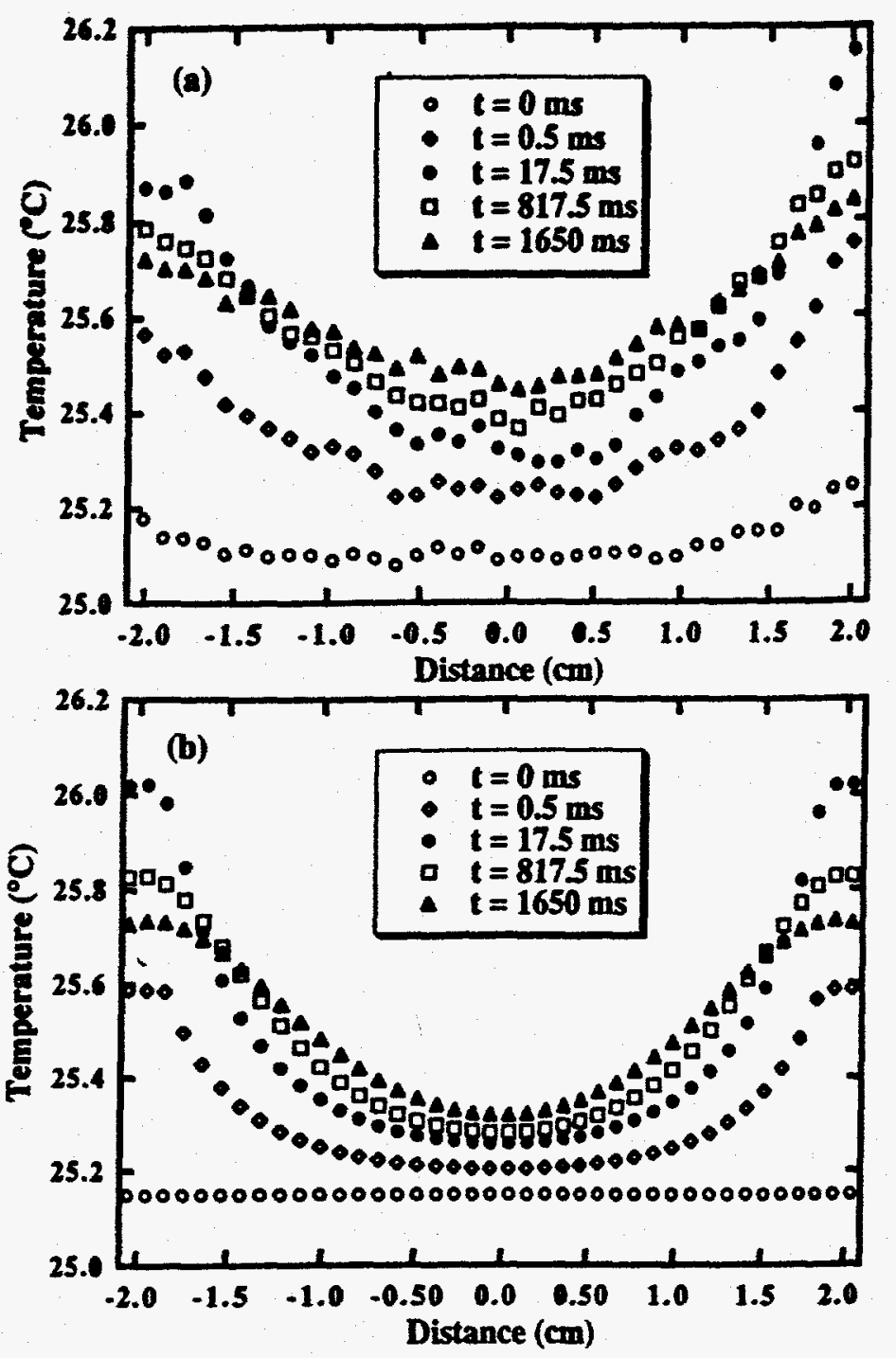

Figure 1: Profiles of the temperature along a varistor diameter before, during, and after a current pulse: (a) measured and (b) simulated.

block, it is logically attributed to the fabrication process. The temperature increases more at the edge of the block because there is a higher current that results from a lower breakdown voltage that can be attributed to a larger grain size. The implied grain size increases monotonically from the center to the edge of the block. Friction in the die used for powder compaction causes density variations that can result in such a systematic variation in the grain size. Moreover, grain growth during sintering can be more rapid in the outer part of the block than in the center of the block. It is difficult to fabricate large ceramic parts with uniform properties, but uniformity is especially important in varistor manufacture because any nonuniformity gets amplified in the conduction process by the electrical nonlinearity. The IR images of varistors cut from large blocks shows that less symmetry is obtained in longer blocks. Current localization tends to be the greatest in highly nonlinear materials, and thus, the production of large varistors requires particularly close control of pressing and sintering operations. These conclusions are consistent with the measured [12] and simulated 
$[6,7]$ data on the energy handling capability of varistor blocks used in distribution-class and station-class arresters.

\section{INTERMEDIATE SCALE}

It is significantly easier to fabricate varistor disks with uniform properties when they are small. However, small varistors exhibit non-uniform current conduction that is of a statistical origin instead of a processing origin. Property variations due to statistical disorder are more significant in small varistors than in large varistors because statistical fluctuations are in general more important in small systems. In small varistors there is a tendency for the current to flow along those conducting paths that have the lowest breakdown voltages because, for example, they are comprised of the largest grains [15]-[18]. Non-uniform heating of small varistors due to current flow along several localized highly conducting paths was observed in Ref. [10]. Since the number of $\mathrm{ZnO}$ grains between the electrodes in the studied samples was only about 40 , a variation of $3-4$ grains can change the current flow in a path by an order of magnitude relative to surrounding paths. Thus, conducting paths with low breakdown voltages carry most of the current and become hotter.

If the number of grains between the varistor electrodes is large enough to apply simple statistics, the difference in the importance of current localization due to statistical disorder in large and small varistors is easy to explain. A grain size distribution characterized by a mean diameter $\bar{d}$ and a variance $\sigma_{d}$ will lead to a differing number of grain boundaries along the various conducting paths that will be approximately Gaussian distributed with a mean $\bar{N}$ and a variance $\sigma_{N}$ given by:

$$
\bar{N}=L / \bar{d} \quad \text { and } \quad \sigma_{N}=\bar{N}^{1 / 2} \sigma_{d} / \bar{d},
$$

where $L$ is the distance between the electrodes. If it is assumed that the breakdown voltage of a current path is just the sum of the breakdown voltage $V_{b}$ of $N$ identical grain boundaries, then the distribution of the breakdown voltage of the various paths is described by a mean $\bar{V}$ and a variance $\sigma_{V}$ that are given by:

$$
\bar{V}=\bar{N} V_{b} \quad \text { and } \quad \sigma_{V}=\bar{N}^{1 / 2} V_{b} \sigma_{d} / \bar{d} .
$$

While the voltage increases in proportion to the number of grain boundaries, the variance of the voltage increases only as the square root of the number of boundaries because smaller grains tend to compensate for larger grains. (In reality, grain boundaries are not identical and the breakdown voltage $V_{b}$ also varies [16]-[18]. However, variations in $V_{b}$ contribute a similar dependence upon $\bar{N}$ to $\bar{V}$ and $\sigma_{V}$ and therefore can be included by just assuming a larger value for $\sigma_{d} / \bar{d}$.) If a varistor is well into the breakdown region where the current $I$ has a nonlinear dependence on the voltage $V$ that can be described by a power law exponent $\alpha$, then the distribution of the current among the possible paths is described by a mean $\bar{I}$ and a variance $\sigma_{I}$. In the simplest approximation,

$$
\bar{I}=I_{0} \bar{V}^{\alpha} \quad \text { and } \quad \sigma_{I}=\alpha \bar{I} \bar{N}^{-1 / 2} \sigma_{d} / \bar{d},
$$

The above classification of the current will be accurate only if the distributions are relatively narrow and approximately Gaussian, but the results agree with the predictions of a more realistic varistor model, at least in a rough way. We examined the computer 
predictions of a "brick model" [1] type of grain structure in which the sizes of the grains (i.e., length in the direction of current flow) were generated by a computer program as random numbers from a log-normal distribution with a given mean and variance. An identical but realistic $I-V$ characteristic was assigned to each of the grain boundaries [6]. Small varistors were modeled as two-dimensional arrays of rectangular grains with $N_{\omega}=100,200$, or 300 grains along the direction perpendicular to the current. All grains were given the same width, but their lengths were randomly generated. The grains were stacked in adjacent columns until a prescribed length corresponding to the thickness of the varistor was reached. Each of the grain boundaries was assumed to have an $I-V$ characteristic with a maximum coefficient of nonlinearity $\alpha=50$. We assumed current flow only in one direction (i.e, along $N_{w}$ available parallel current paths containing a varying number of grain boundaries). The current distribution in the sample was found by just calculating separately the current in each path. The general predictions of this simple model agree with those of more realistic Voronoi network models [15]. The current localized in the paths with the least number of grain boundaries, or grains. The localization is particularly pronounced in the nonlinear region of the $I-V$ characteristic. And the maximum current localization occurs at the voltage for which the sample has the maximum nonlinearity coefficient. In Fig. 2, values of $\sigma_{I} / \bar{I}$ found from this model are compared to the predictions of Eq. (3) for different values of $\sigma_{d} / \bar{d}$. The agreement is good, and it improves as $\bar{N}$ becomes large or $\sigma_{\alpha}$ becomes smaller.

Current localization on highly conducting paths will be negligible if the variance of the current distribution is small relative to its mean, or when $\sigma_{I} / \bar{I} \ll 1$. This condition together with Eq. (3) leads to a criterion for the number of grain boundaries required in a varistor in which current localization due to statistical variations in grain size will be unimportant:

$$
\bar{N} \gg \alpha^{2}\left(\sigma_{d} / \bar{d}\right)^{2} .
$$

If it is assumed, for example, that $\sigma_{d} / \bar{d}=0.1$ and $\alpha=50$, then $\bar{N} \gg 25$ should result in minimal current localization due to grain size variations. (Note the strong influence of nonlinearity exponent.)

Large arrester blocks contain thousands of barriers in each path, so current localization due to statistical disorder of the grain sizes is too small to cause a failure by puncture or thermal cracking. Based on the results of a computer simulation of non-uniform Joule heating in disordered networks, Vojta and Clarke [13] have recently proposed that puncture failures have microstructural origin. However, our present results indicate that their conclusions are not valid for large (high voltage) varistor blocks. In large varistors, destructive failures are caused by macroscopic nonuniformities due to processing (e.g., compaction and sintering) $[6,7]$. Varistors for electronic applications are another matter. Because they may have fewer than 10 barriers in a current path, current localization due to grain size variations can be severe.

A formula that goes beyond inequality (4) to describe the influence of varistor size on current localization due to statistical disorder can be obtained if it is assumed that the breakdown voltages of the various conducting paths have a Gaussian distribution with a mean and variance given by Eq. (2). Then, well into the breakdown region, the current distribution on these paths is

$$
p(i)=\frac{i^{-(1-1 / \alpha)}}{\alpha \sigma_{V} \sqrt{2 \pi}} \exp \left[-\frac{\bar{V}^{2}}{2 \sigma_{V}^{2}}\left(1-i^{1 / \alpha}\right)^{2}\right]
$$



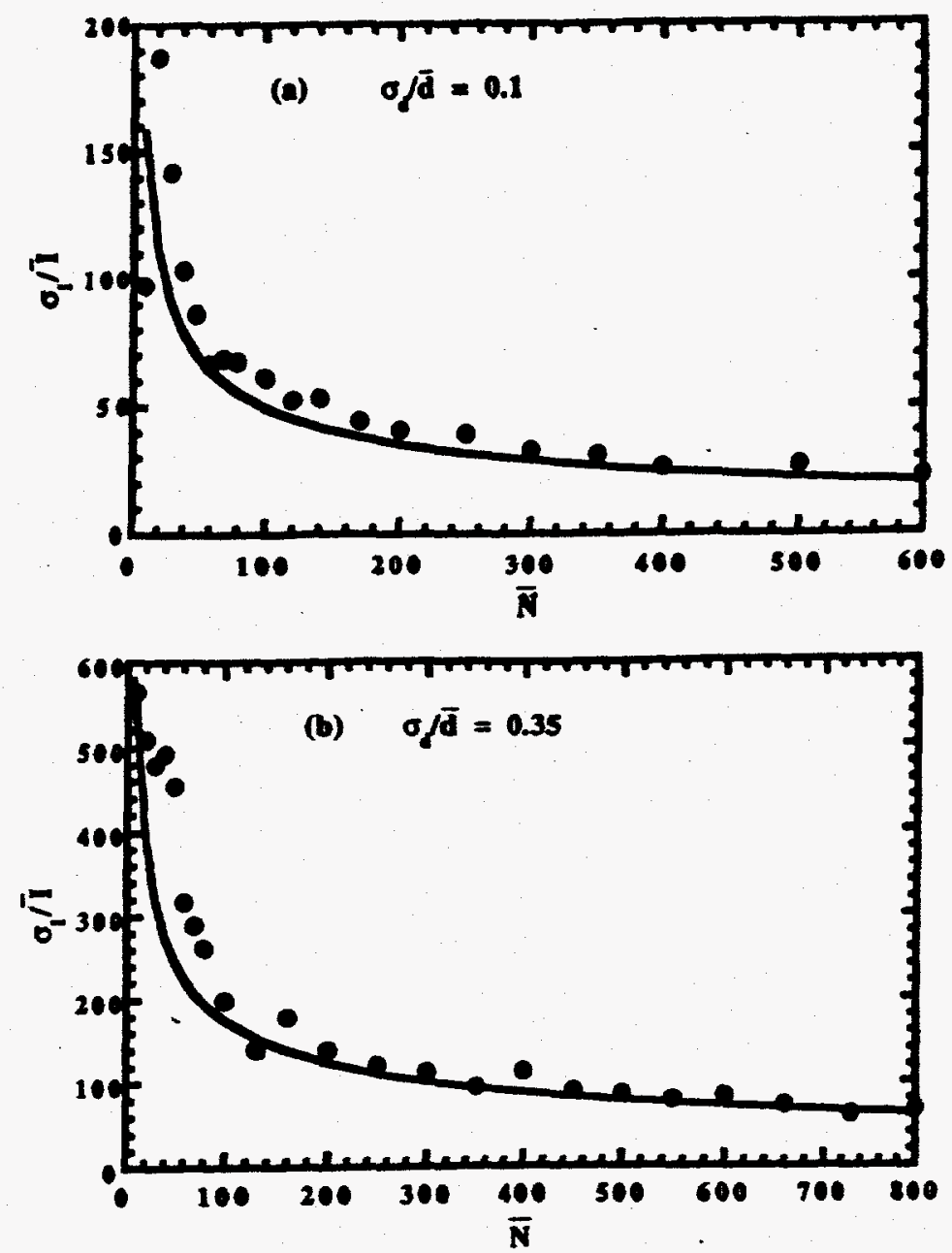

Figure 2: Relative variance $\sigma_{I} / \bar{I}$ calculated from Eq. (3) (solid lines) and simulated within the disordered "brick model" (full circles) as a function of the average number of grains between the electrodes $\bar{N}$, for $\sigma_{d} / \bar{d}=(\mathrm{a}) 0.1$ and (b) 0.35 .

where $i$ is defined as $i=I / I=I /\left(I_{0} \bar{V}^{a}\right)$. The current distribution $p(i)$ is asymmetric, and, depending on the values of $\alpha$ and $\sigma_{V} / \bar{V}$, can have a long tail for large currents. The presence of such a long tail corresponds to (and describes analytically) the current localization phenomenon. A current path gets hot because of current localization and Joule heating only if it carries more current than an average path by some factor which can be denoted as $n$ :

$$
I_{h p} \geq n\langle I\rangle \text {, }
$$

where $n$ is a rather arbitrary number between about 2 and 5 depending upon the choice of criterion for "hot". $\langle I\rangle$ in Eq. (6) is the actual expectation value of the distribution (5),

$$
\langle I\rangle=I_{0} \bar{V}^{\alpha}\langle i\rangle=I_{0} \bar{V}^{a} \int_{0}^{\infty} i p(i) d i,
$$

which in general is not equal to $\bar{I}$ from the rough approximation in Eq. (3). The fractional number of hot paths $f_{h p}$ in a varistor sample for a given factor $n$ is calculated as the integral 


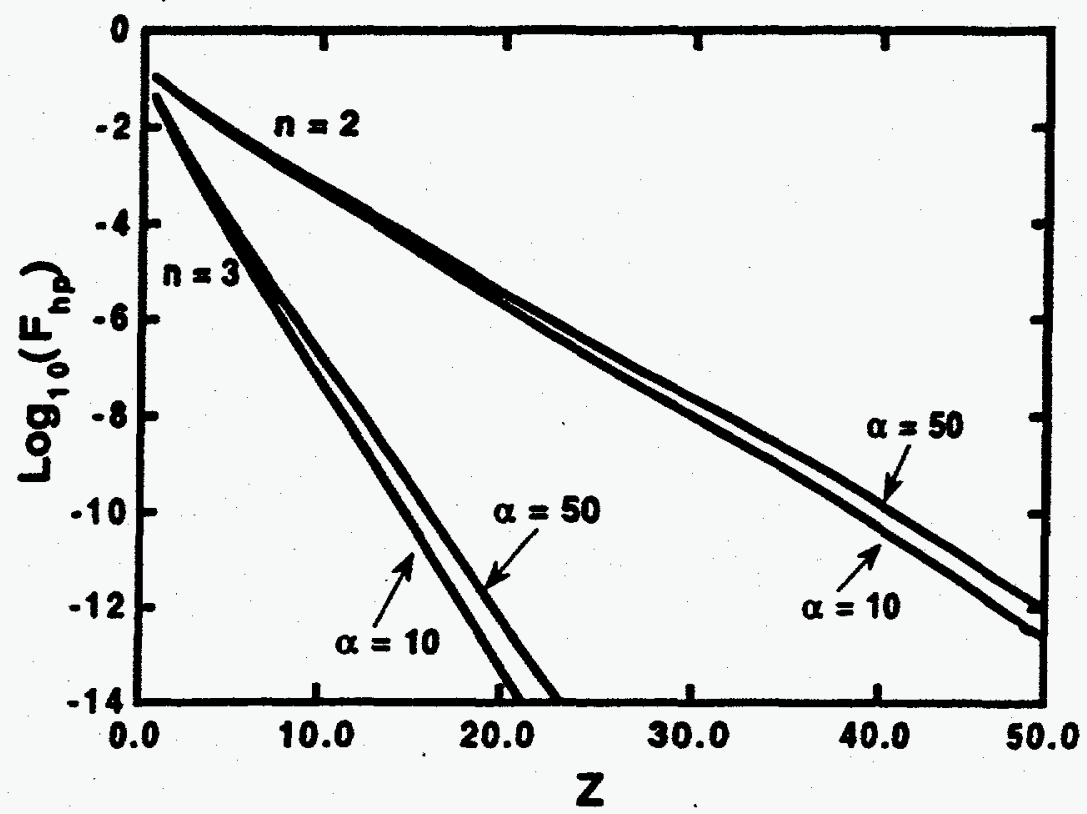

Figure 3: Fractional number of hot paths as a function of $z=\frac{\bar{N}}{2 \alpha^{2}}\left(\frac{\bar{d}}{\sigma_{d}}\right)^{2}$. Since $z$ itself depends on $\alpha$, the fractional number of hot paths is a very strong function of $\alpha$. The slightly different curves for $\alpha=10$ and $\alpha=50$ show that the single dependance on $z$ is not perfect.

describing the number of paths in the high current tail of the distribution:

$$
f_{h p}=\int_{n(i)}^{\infty} p(i) d i=\sqrt{\frac{z}{\pi}} \int_{n(i)}^{\infty} i^{1 / \alpha-1} \exp \left[-z \alpha^{2}\left(i^{1 / \alpha}-1\right)^{2}\right] d i,
$$

where

$$
z=\frac{\bar{N}}{2 \alpha^{2}}\left(\frac{\bar{d}}{\sigma_{d}}\right)^{2}
$$

Computed numerically logarithm of the fractional number of hot paths as a function of the parameter $z$ is presented in Fig. 3 for $n=2$ and 3 , and for $\alpha=10$ and 50 . It is seen, for example, that for varistors with $z>5$ only less than one in about $10^{4}$ paths conducts current 3 times $(n=3)$ higher than the average, which is still not the case of a very hot path. The choice of $z$ as an independent variable in Eq. (8) and in Fig. 3 leads to an approximate scaling of the results, so that they appear as only weakly dependent on $\alpha$. This, however, does not mean that $f_{h p}$ is not sensitive to the values of $\alpha$. On the contrary, since $z$ itself is a strongly varying function of $\alpha$ [Eq. (9)], so is $f_{\text {hp }}$.

The predictions of Eq. (8) for small and large varistors contrast remarkably. A typical varistor for electronic applications is a small disk of about $1 \mathrm{~cm}$ diameter and $0.1 \mathrm{~cm}$ thickness. Typically, the grains of such a varistor are described by $\bar{d}=100 \mu \mathrm{m}$ and $\sigma_{d} / \bar{d}=0.1$, and the nonlinearity coefficient might be $\alpha=25$. If the hot paths are assumed to be those paths that carry a current $I>2\langle I\rangle$ (i.e., $n=2$ ), then the parameter $z$ of Eq. (9) is 0.8 , and $f_{h p} \approx 0.1$. Hence, current localization is predicted to be a problem. Commercial varistors for electronic applications have rather low nonlinearity coefficient, although it is not difficult to produce small varistors having an $\alpha$ of 50 or more. However, increase of $\alpha$, according to Eq. (9), would lead to smaller values of $z$, and [by Eq. (8)] to an even higher probability of hot paths, thus making the varistor even more prone to failure. 
On the other hand, a distribution-class arrester block is typically a disk of about $4 \mathrm{~cm}$ diameter and $4 \mathrm{~cm}$ thickness. The smaller grains of such a varistor block can be described by $\bar{d}=20 \mu \mathrm{m}$ and $\sigma_{d} / \bar{d}=0.1$, and the nonlinearity coefficient of such a varistor might be $\alpha=50$. Again, if the hot paths are assumed to be those paths carrying a current $I>2\langle I\rangle$ (which is a very conservative definition), then the parameter $z$ of Eq. (9) is about 40 , and $f_{h p} \approx 10^{-10}$. So there will be no hot paths due to statistical disorder in such blocks, even though the total number of available current paths exceeds a million. Clearly, current localization resulting from statistical disorder is only important in small varistors.

\section{MICROSCOPIC SCALE}

In Ref. [10], varistor heating was examined at the microscopic level in thin slices cut from low voltage varistors that have grain sizes over $100 \mu \mathrm{m}$. These slices were polished to about $100 \mu \mathrm{m}$ thick, so that electrical conduction was essentially confined to a single two-dimensional layer of grains. The thermal images of the slices taken during $1 \mathrm{~ms}$ long high current density pulses showed distinct conducting paths very similar to those obtained from computer simulations of the current flow in a varistor Voronoi network [15]-[17]. This striking similarity substantiate the theoretical calculations and confirm modeling predictions. The paths in the thermal images consisted of chains of localized hot spots that mark the grain boundaries - occurring where the potential is dropped across Schottky-type barriers and the heat is generated. Local temperatures at the grain boundaries were found to rise well above that of the interiors of the grains during $1 \mathrm{~ms}$ current pulses of about $300 \mathrm{~A} / \mathrm{cm}^{2}$. The images of hot spots were similar to photographs of the electroluminescence from grain boundaries [19]-[21]. These observations clearly showed that varistor heating is localized at the grain boundaries during electrical breakdown.

The fine spatial resolution of the IR camera allows the temperature profile along individual conducting paths to be resolved. Figure 4(a) shows one such profile measured along the line through the centers of two distinct hot spots in the IR image of a slice cut from low voltage varistor. These spots were chosen for the purpose of clarity, although the size of the grain between them (about $200 \mu \mathrm{m}$ ), was larger than the average grain size. The profile shows two clear peaks corresponding to grain boundaries, and the difference between the temperature at the grain boundaries and the lowest temperature in the grain interiors is about $15-20^{\circ} \mathrm{C}$. In order to interpret the processes that lead to the observed temperature profile, a simple model of the heating was employed. Since the important heat transfer processes take place along conducting paths, a one-dimensional heat diffusion equation of the following form was used:

$$
c D \frac{\partial^{2} T(x, t)}{\partial x^{2}}-c \frac{\partial T(x, t)}{\partial t}+Q(x, t)-H T(x, t)=0,
$$

where $c=3.3 \mathrm{~J} /\left(\mathrm{cm}^{3} \mathrm{~K}\right)$ is the heat capacity, $D=0.1 \mathrm{~cm}^{2} / \mathrm{s}$ is the thermal diffusivity of the material, and the term $-H T(x, t)$ describes the loss of heat from the grains in a path. The time-dependent temperature $T(x, t)$ at the distance $x$ from the grain boundary is measured relative to the sample temperature at $t=0$, taken as $20^{\circ} \mathrm{C}$. The grain size $d$ is taken as 200 $\mu \mathrm{m}$, in accordance with the particular measurement shown in Fig. 4(a). In the prebreakdown and in the nonlinear region of the varistor $I-V$ characteristic, almost all of the voltage drop appears across the grain boundaries, so that vast majority of the energy goes into heating the grain boundaries. Therefore, for simplicity, it is assumed that the heat is generated 

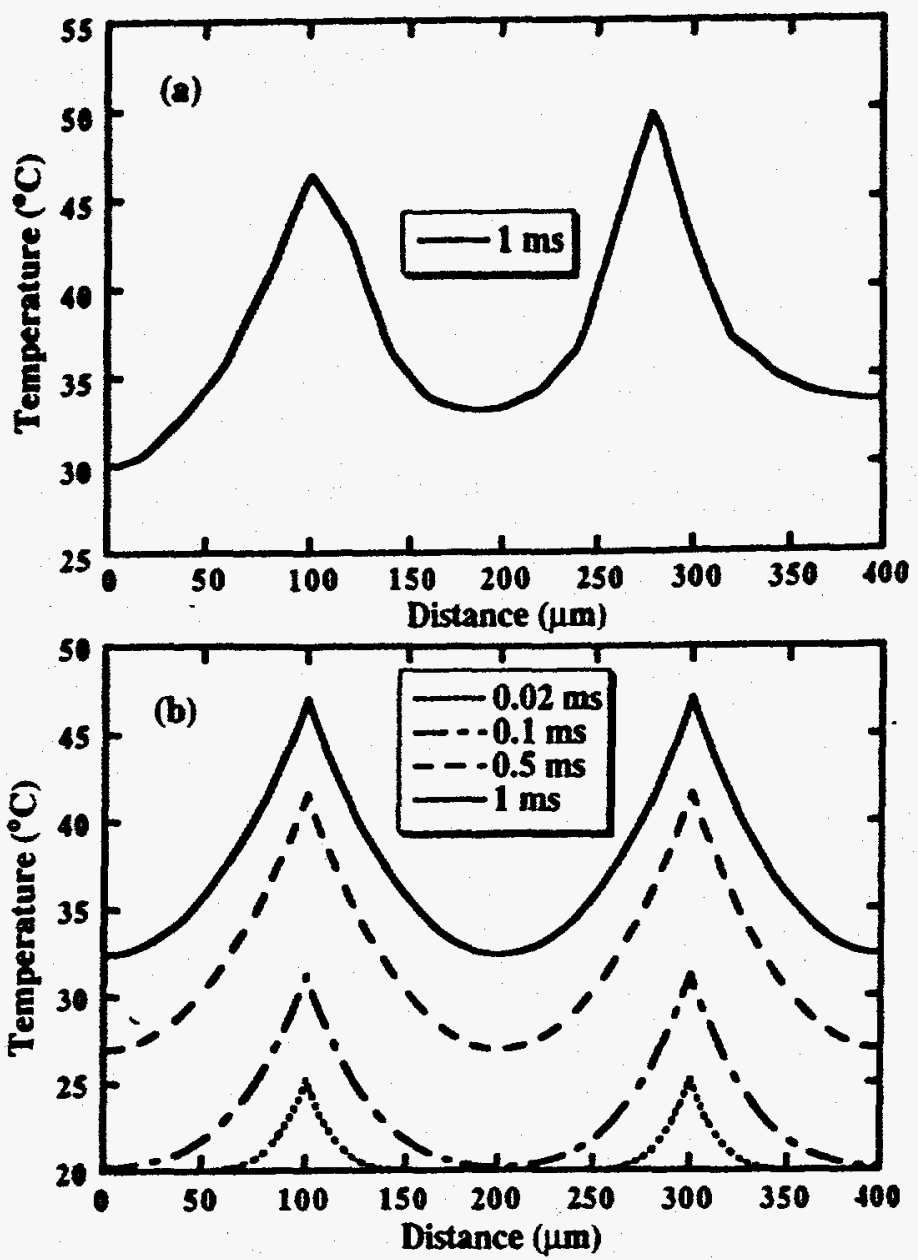

Figure 4: (a) Temperature profile along the line between two bright spots in an IR image of a thin varistor slice. (b) Time-dependent temperature profiles obtained from the solution of the corresponding heat transfer equation.

only at the grain boundaries. Since the grain boundary region is very narrow $(\sim 1000 \AA)$ compared to the grain size, it is reasonable to approximate the spatial dependence of the power input $Q(x, t)$ in Eq. (10) by the Dirac delta function. The time dependence of $Q(x, t)$ is taken as a constant pulse that starts at $t=0$, so that $Q(x, t)=q \theta(t) \delta(x)$, where $\theta(t)$ is the unit step function. The input power $q$ at the surface of the grain boundaries can be estimated as follows. The voltage across the sample is $80 \mathrm{~V}$, and the total current is about 1.5 A. However, the current flows mainly along a number of conducting paths. Judging from the IR image of the whole slice, about $10 \%$ of the total current flew along the particular path considered here. Finally, taking into account the length $(l=5.5 \mathrm{~mm})$, the width $(w=5 \mathrm{~mm})$, and the thickness $(h \approx 0.1 \mathrm{~mm})$ of the sample, we obtain a power input $q$ of about $2200 \mathrm{~W} / \mathrm{cm}^{2}$. Heat diffuses not only along the line $x$, as is explicitly described by Eq. (10), but it dissipates in directions perpendicular to the current flow to the grains adjacent to the conducting path, to the substrate beneath the varistor sample, and to some extent to the air above the sample. All of this heat dissipation is described by the single sink term $-H T(x, t)$ in Eq. (10), which assumes that the heat loss at position $x$ and time 
$t$ is proportional to the temperature rise along the conducting path $T(x, t)$. The parameter $H$ can be estimated simply by assuming that the heat dissipates by conduction to adjacent grains and to a substrate with thermal properties that are not much different from those of zinc oxide. In this particular case, $H=2 c D /(h d)+c D / d^{2} \approx 4100 \mathrm{~W} /\left(\mathrm{cm}^{3} \mathrm{~K}\right)$, where the first term corresponds to the heat dissipation to adjacent grains, and the second term describes the amount of heat dissipated to the substrate. Since there is also a little heat escaping to the air, we just assume $H=5000 \mathrm{~W} /\left(\mathrm{cm}^{3} \mathrm{~K}\right)$. The boundary conditions that have to be satisfied by the solution of Eq. (10) are

$$
T(-d / 2, t)=T(d / 2, t) \quad \text { and }\left.\quad \frac{\partial T(x, t)}{\partial x}\right|_{x=d / 2}=\left.\frac{\partial T(x, t)}{\partial x}\right|_{x=-d / 2}=0 .
$$

These conditions ensure that the temperature profile is symmetric around the grain boundary, and that there is no heat transfer along the $x$ direction at the center of the grain. Eq. (10) can be solved analytically using Laplace transforms, and the solution can be written as

$$
T(x, t)=\frac{q}{H d}\left(1-e^{-H t / c}\right)+\frac{2 q}{c d} \sum_{n=1}^{\infty} \frac{\left(1-e^{-A_{n} t}\right)}{A_{n}} \cos (2 \pi n x / d)
$$

where

$$
A_{n}=\frac{H}{c}+\frac{4 \pi^{2} n^{2} D}{d^{2}} .
$$

The series in Eq. (12) converges relatively fast and can be calculated numerically. The resulting temperature profiles for $t=0.02 \mathrm{~ms}, 0.1 \mathrm{~ms}, 0.5 \mathrm{~ms}$, and $1 \mathrm{~ms}$ are shown in Fig. 4(b). The final temperature profile at the end of a 1 ms current pulse, shown in Fig. 4(b) as the solid curve, is in excellent agreement with that in Fig. 4(a), measured in the experiment. The calculated data also show that at the beginning of the current pulse the temperature rises fast only at the grain boundaries and in the immediate neighborhood of the grain boundaries. But as the pulse continues and the heat diffuses into the grain interiors, the temperature of the grain boundaries and grain interiors increase at nearly the same rate. This is consistent with the experimental observations and has some significant implications. Varistors exhibit a rather surprising increase in their energy handling capability at very high currents. This was first noticed by Sakshaug, Burke, and Kresge [11], who attributed this increase to a change on a microscopic scale (i.e., the scale of the grain size) in the temperature distribution that occurs at high current. In contrast to currents in the prebreakdown or the nonlinear region of the $I-V$ characteristic, where the voltage drop appears almost exclusively across the grain boundaries, the current is so high when a varistor operates in the upturn region that the voltage drop inside the grains becomes significant, and the heating becomes more uniformly distributed. This, according to the interpretation of Ref. [11], should lead to reduced temperature difference between grain interiors and grain boundaries, thereby reducing the thermal shock and leading to higher energy handling capability. However, as discussed above, the difference between the temperature at the grain boundary and the grain interior quickly reaches an essentially steady-state value, and it never becomes very large - maybe only about $35^{\circ} \mathrm{C}$ for severe current pulses. This means that the heat transfer is too fast to permit temperature differences on a scale of the grain size that could cause failure by generating thermal stresses. We conclude that varistor failures are not caused by temperature differences on the microscopic scale. 
The heating of $\mathrm{ZnO}$ varistors by electrical pulses over a range of spatial scales that extends from the microscopic to the macroscopic has been discussed. Non-uniform heating due to current localization was shown to have different origins and different consequences for device failure in large and small varistors. On a macroscopic scale, heating has been examined in arrester blocks of a distribution-class size, and non-uniform temperature distributions have been attributed to inhomogeneities which originate during ceramic processing.

In small varistors for electronic applications, non-uniform heating due to current localization appears on a finer spatial scale. Localized conducting paths became important when the number of grains in the electrical paths decreased to the point where statistical variations in the electrical breakdown voltage became significant. It is found that influence of statistical disorder due to grain size and barrier hight variations changes with varistor size in a manner that can be described with relatively simple mathematics.

At the microscopic level, the heating is shown to take place at grain boundaries. Theoretical modeling has confirmed the experimental observation that local temperature increases of up to $20^{\circ} \mathrm{C}$ above the grain interiors during varistor breakdown. These results imply that current localization on the size scale of the grains does not cause failures.

\section{ACKNOWLEDGEMENTS}

I thank G.D. Mahan, F. Modine and H. Wang for valuable discussions. This work was supported by the U.S. Department of Energy through the Assistant Secretary for Energy Efficiency and Renewable Energy, Office of Transportation Technologies, as part of the High Temperature Materials Laboratory User Program and by the Office of Basic Energy Science under contract DE-AC05-96OR22464, managed by Lockheed Martin Energy Research Corporation.

\section{References}

[1] L.M. Levinson and H.R. Philipp, Ceram. Bull. 65, 639 (1986).

[2] T.K. Gupta, J. Am. Ceram. Soc. 73, 1817 (1990).

[3] K. Eda, J. Appl. Phys. 56, 2948 (1984).

[4] M.V. Lat, IEEE Trans. Power Appar. Syst. 102, 2194 (1983).

[5] S. Horiguchi, F. Ichikawa, A. Mizukoshi, K. Kurita and S. Shirakawa, IEEE Trans. Power Delivery 3, 1666 (1988).

[6] M. Bartkowiak, M.G. Comber and G.D. Mahan, J. Appl. Phys. 79, 8629 (1996).

[7] M. Bartkowiak, M.G. Comber and G.D. Mahan, accepted for publication in IEEE Trans. Power Delivery.

[8] G. Hohenberger, G. Tomandl, R. Ebert, and T. Taube, J. Am. Ceram. Soc. 74, 2067 (1991). 
[9] A. Mizukoshi, J. Ozawa, S. Shirakawa, and K. Nakano, IEEE Trans. Power Appar. Syst. 102, 1384 (1983).

[10] H. Wang, M. Bartkowiak, F.A. Modine, R.B. Dinwiddie, L.A. Boatner, and G.D. Mahan, accepted for publication in J. Am. Ceram. Soc.

[11] E.C. Sakshaug, J.J. Burke, and J.S. Kresge, IEEE Trans. Power Delivery 4, 2076 (1989).

[12] K.G. Ringler, P. Kirkby, C.C. Erven, M.V. Lat, and T.A. Malkiewicz, IEEE Trans. Power Delivery 12, 203 (1997).

[13] A. Vojta and D.R. Clarke, J. Appl. Phys. 81, 985 (1997).

[14] Z.-C. Cao, R.-J. Wu, and R.-S. Song, Mater. Sci. and Engr. B 22, 261 (1994).

[15] M. Bartkowiak and G.D. Mahan, Phys. Rev. B 5110825 (1995).

[16] M. Bartkowiak, G.D. Mahan, F.A. Modine, and M.A. Alim, J. Appl. Phys. 79, 273 (1996).

[17] M. Bartkowiak, G.D. Mahan, F.A. Modine, M.A. Alim, R.J. Lauf, and A.D. McMillan, J. Appl. Phys. 80, 6516 (1996).

[18] C.-W. Nan and D. Clarke, J. Am. Ceram. Soc. 79, 3189 (1996).

[19] G.E. Pike, S.R. Kurtz, P.L. Gourley, H.R. Phillips, and L.M. Levinson, J. Appl. Phys. 57, 1552 (1985).

[20] M. Rossinelli, G. Blatter, and F. Greuter, British Ceram. Proc. 36, 1 (1985).

[21] F. Greuter, G. Blatter, M. Rossinelli, and F. Stucki, Ceramic Trans. 3, 31 (1989). 